\title{
Programa de intervención para promover la comunicación sobre sexualidad en padres de hijos con discapacidad intelectual ${ }^{1}$
}

\author{
Intervention program to promote communication \\ about sexuality in parents of children \\ with intellectual disability
}

\author{
Susana Xóchitl Bárcena Gaona, Yolanda Guevara Benitez \\ y María Vianey Rodríguez Gutiérrez²
}

Citación: Bárcena G., S.X., Guevara B., Y. y Rodríguez G., M.V. (2020). Programa de intervención para promover la comunicación sobre sexualidad en padres de hijos con discapacidad intelectual. Psicología y Salud, 30(2), 173-187. https://doi.org/10.25009/pys.v30i2.2652.

RESUMEN

\begin{abstract}
El objetivo del presente estudio fue evaluar la efectividad de un programa dirigido a padres de jóvenes con discapacidad intelectual para promover la comunicación con sus hijos sobre temas relativos a la sexualidad. El programa se diseñó con fundamento en los conceptos de autoeficacia y aprendizaje estructurado. Participaron cinco padres, cuya edad promedio fue de 43 años, con hijos con síndrome de Down o con otro tipo de discapacidad intelectual, con edad promedio de 18 años. Se utilizó un diseño $\mathrm{n}=1$ y se recabó información antes de la intervención, al finalizar ésta y transcurridos tres meses, sobre las variables de autoeficacia, expectativas y comunicación sobre sexualidad. El programa de intervención tuvo una duración de diez sesiones. Los resultados indican que, desde la fase de pretest, la mayoría de los participantes reportaron tener expectativas positivas hacia la comunicación; después de la intervención, todos ellos reconocieron los efectos positivos de la comunicación. Resultados similares se obtuvieron en cuanto a la autoeficacia percibida para hablar con sus hijos sobre todos los temas de sexualidad incorporados al programa. Los mayores efectos se observaron en el número de temas abordados porque se logró que, en la fase de seguimiento, todos los padres mantuvieran comunicación con sus hijos sobre todos los temas abordados en el taller. También se observó un aumento en el número de habilidades conductuales desarrolladas por los hijos de los participantes. Al tiempo que se concluye que el programa fue eficaz para lograr su objetivo, se discuten las aportaciones y limitaciones del estudio.
\end{abstract}

Palabras clave: Sexualidad; Discapacidad; Comunicación; Padres; Programa de intervención.

\begin{abstract}
The objective of this study was to assess the effectiveness of a program aimed to parents of young people with intellectual disabilities, in order to promote communication with their children about sexuality issues. The program was designed based on the concepts of self-efficacy and structured learning. Five parents participated, whose average age was 43 years, three of whom had children with Down syndrome, and the rest with another type of intellectual disability, with an average age
\end{abstract}

\footnotetext{
${ }^{1}$ Estudio Financiado por el Programa de Apoyo a Proyectos de Investigación e Innovación Tecnológica (PAPIIT) de la Universidad Nacional Autónoma de México, clave 300416. Artículo recibido el 15 de enero y aceptado el 24 de mayo de 2019.

${ }^{2}$ Unidad de Investigación Interdisciplinaria en Ciencias de la Salud y la Educación, Primer piso, Cubículo 8, Facultad de Estudios Superiores Iztacala, Avenida de los Barrios 1, Col. Los Reyes Iztacala, 54090 Tlalnepantla, Edo. de México, México, correos electrónicos: s.barcena@unam.mx,yolaguevara@hotmail.com y vianbutterfly15@gmail.com.
} 
of 18 years. A design $n=1$ was used, and data about self-efficacy, expectations, and communication about sexuality variables were collected before, at the end of the intervention this, and after three months. The intervention program lasted ten sessions. The results indicate that, from the pretest phase, most of the participants reported having positive expectations toward the communication, and after the intervention, all parents recognized the positive effects of the said communication. Similar results were obtained regarding the self-effcacy perception to talk with their children about all sexuality issues, incorporated into the program. The greatest effects were observed in the number of topics addressed, because all parents were able to communicate with their children about all the topics included in the workshop. There was also an increase in the number of behavioral skills developed by the children. It is concluded that the program was effective to achieve its objectives. The contributions and limitations of the study are discussed.

Key words: Sexuality; Disability; Communication; Parents; Intervention program.

$\mathrm{A}$ lo largo de su vida, los seres humanos muestran cambios biológicos, cognoscitivos, emocionales y conductuales que van marcando sus períodos evolutivos. Según diversos autores $(\mathrm{Ca}-$ ricote, 2012; Guevara, Robles, Aguilar y Luna, 2016), es durante la adolescencia y la primera juventud cuando aparecen los mayores cambios en la vida, y es por ello que los jóvenes se encuentran en situaciones de mayor riesgo durante esos periodos.

La Encuesta Nacional de Salud (ENSANUT 2015), realizada por el Instituto Nacional de Salud Pública de México y la Organización Mundial de la Salud (OMS) (2016), muestran un cuadro de múltiples riesgos en la vida de los jóvenes y adolescentes, como los altos índices de contagio y mortalidad por diversas infecciones de transmisión sexual (ITS), particularmente por el virus de inmunodeficiencia humana (VIH), así como de embarazos adolescentes, abortos y partos precoces. Estos problemas están directamente asociados a prácticas sexuales inapropiadas o de riesgo. En la literatura sobre la sexualidad hay coincidencias de que muchos de los problemas de salud de adolescentes y jóvenes pueden prevenirse mediante una educación sexual que les permita conocer cómo protegerse y cómo vivir su sexualidad de manera sana y de un modo acorde a su edad.

La educación sexual debe considerar la prevención del abuso sexual, especialmente en poblaciones vulnerables, como niños, adolescentes y personas con discapacidad. Como apunta Pereda (2016), diferentes metaanálisis llevados a cabo en años recientes en muestras poblacionales de 24 países dan cuenta de la extensión de la victimización sexual de niños y jóvenes; los datos confirman que $8 \%$ de la población masculina y $20 \%$ de la femenina han sido víctimas de algún tipo de abuso sexual, por lo que se considera este fenómeno como un problema mundial. En México, poco se sabe de la extensión del abuso sexual infantil dada la carencia de estudios sistemáticos, pero el Sistema Nacional para el Desarrollo Integral de la Familia (DIF), (cfr. García, Loredo, Trejo y Casas, 2013) reportó en 2010 que anualmente se reciben 900 denuncias por ese delito.

Nosek, Foley, Hughes y Howland (2001) exponen una serie de razones por las cuales las personas con discapacidad son más vulnerables ante el abuso: una mayor dependencia hacia los demás, condiciones de sobreprotección y aislamiento social, mayor riesgo de manipulación por parte de otros (incluyendo a sus cuidadores), negación de sus derechos humanos, desvalorización social hacia ellas, baja autoestima, percepción de impotencia y mínima o nula educación sobre prácticas adecuadas e inadecuadas de la sexualidad. Por su parte, Giménez, Gil, Ruiz y Díaz (2017) señalan que el abuso sexual afecta de un modo especial a las personas con discapacidad, y por ello el rol de los profesionales resulta ser esencial para identificar y tratar los problemas asociados; dichos autores también subrayan la necesidad de enfocar el trabajo profesional en la prevención de este problema.

La definición de abuso sexual infantil incluye "cualquier solicitud o ejercicio de contacto, caricias, juegos o toqueteos, en los que al menos uno de los implicados no desea, conoce o carece de conciencia de lo que está pasando, y que se obtiene por la fuerza o la ascendencia sobre la víctima" (Rodríguez, Aguilar y García, 2012, p. 59). Vivir con discapacidad implica que la persona con una condición limitante (motriz, intelectual o sensorial) puede enfrentar barreras impuestas por su 
entorno social, las cuales se traducen como limitaciones para su inclusión educativa, familiar, comunitaria, cultural, social y de atención a la salud.

Todo ello coloca a esta población en una situación de alto riesgo de sufrir abuso sexual, enfermedades de transmisión sexual (ITS), VIH/sida y embarazos no deseados, como lo demuestran diversas investigaciones hechas con poblaciones de distintos países, México incluido (Giménez et al., 2017; Guevara, Robles, Rodríguez y Frías, 2016; Morell, Gil, Díaz y Caballero, 2017; Nosek et al., 2001; Robles y Guevara, 2017; Smith, 2008).

Es importante destacar que las personas con discapacidad muestran el mismo interés por participar en las actividades que realizan sus pares durante su adolescencia, y tienen las mismas inquietudes sobre cómo establecer relaciones interpersonales. Sin embargo, esta cuestión genera controversia entre sus familiares, e incluso entre algunos educadores (Rivera, 2008). En una investigación realizada por Díaz, Gil, Ballester, Morell y Molero (2014) en adultos con discapacidad intelectual, la mayoría declaró haber sentido atracción hacia algunas personas, tenido por lo menos una pareja en su vida y compartido besos y caricias con otras. Asimismo, más de la mitad afirmó haber tenido fantasías sexuales y al menos una relación sexual. Kijak (2013), por ejemplo, encontró que los adultos con discapacidad intelectual tienen experiencias sexuales, aunque sus conocimientos sobre la sexualidad son deficientes; recurren a la masturbación y a las caricias para expresar sus necesidades sexuales, pero la falta de información sobre cómo llevar a cabo estas prácticas autoeróticas tiene como consecuencia que las ejecuten en forma inapropiada y peligrosa -como practicarlas en lugares públicos o utilizando objetos-, con el riesgo de lastimarse. Por ello, es importante diseñar programas de intervención para esta población considerando sus necesidades afectivas y sexuales y no únicamente la prevención del abuso sexual.

Aunado a lo anterior, las personas con discapacidad intelectual suelen estar sobreprotegidas por sus padres $u$ otros adultos, quienes las limitan personal y socialmente. Los padres no escuchan sus opiniones y deseos, y por ello las formas de que disponen para expresar su sexualidad se ven drásticamente limitadas (Caricote, 2012). En un estudio realizado por Björnsdóttir, Stefánsdóttir y
Valgerður (2017) se encontró que los individuos con discapacidad intelectual no se perciben como seres autónomos, pues ni siquiera tienen la libertad de tomar decisiones sencillas, como las horas de comer o de dormir, por ejemplo. Además, la mayor parte del tiempo están acompañados por quienes se encargan de su cuidado, lo que limita su autonomía y privacidad. Esto es importante si se considera que la mayoría de las personas conocen a sus parejas potenciales en la escuela, el trabajo o los espacios sociales, pero esas oportunidades se ven gravemente limitadas cuando se trata de personas discapacitadas, quienes por lo regular no tienen acceso a esos espacios debido a las barreras sociales y familiares (Shakespeare, 2000). Así, ante el panorama expuesto, quienes sufren de discapacidad tienen más dificultades para establecer relaciones de amistad y así encontrar una pareja, en comparación con las personas sin esa discapacidad (Howland y Rintala, 2001).

La educación sexual de estas personas es una necesidad urgente porque se requiere brindarles las herramientas para cuidar su salud, así como para acabar con diversos mitos y estereotipos acerca de ellas, como por ejemplo su reducida o nula sexualidad. Estas actitudes negativas constituyen uno de los principales impedimentos para el desarrollo psicosexual de esta población (Insa, 2005; Morell et al., 2017). De hecho, privarla de educación sexual integral implica, como señala Caricote (2012), un crimen y un atentado a sus derechos humanos.

La literatura especializada demuestra consistentemente que cuando los jóvenes reciben educación sexual retardan el inicio de su vida sexual, y que cuando la inician es más probable que utilicen el preservativo (Schaalma, Abraham, Gilmore y Kok, 2004); sin embargo, las personas discapacitadas difícilmente reciben dicha educación porque los programas orientados a la promoción de la salud y la prevención de riesgos no están diseñados para ellas (Organización Mundial de la Salud, 2015), pues se les considera innecesarios (Rivera, 2008).

Por estos motivos, los padres desempeñan un papel esencial en la promoción de conductas sexuales saludables de sus hijos cuando establecen con ellos una comunicación adecuada (Bárcena, Robles y Díaz-Loving, 2013). Los estudios efec- 
tuados en la población no discapacitada demuestran que entre mayor es el grado de comunicación sobre la sexualidad entre padres e hijos, menores probabilidades hay de que estos últimos se involucren en conductas sexuales de riesgo (Markham et al., 2010) y sean víctimas de abuso sexual (Chen, Dunne y Han, 2007). Por el contrario, cuando los padres no hablan con sus hijos acerca de esos tópicos, propician que estos adquieran una información distorsionada a través de sus pares, la televisión y la Internet (Caricote, 2008).

De acuerdo con Eisenberg, Sieving, Bearinger, Swain y Resnick (2006), para que los padres tomen la decisión de hablar sobre sexualidad con sus hijos es fundamental que estén convencidos de la necesidad de hacerlo; por lo general, comienzan a abordar con sus hijos esos temas cuando aparecen en ellos los primeros cambios físicos o cuando logran tener sus primeras citas románticas. En el caso de los padres de hijos con discapacidad, es probable que no crean conveniente abordar temas sexuales con ellos al desestimar su capacidad para establecer relaciones sexuales y afectivas.

Entre los modelos explicativos utilizados para investigar las variables que intervienen para que los padres establezcan diálogos sobre la sexualidad con sus hijos destaca el propuesto por Dilorio et al. (2001), modelo que resulta muy adecuado porque está fundamentado en los conceptos de autoeficacia -definida como la creencia de una persona en su propia capacidad para realizar una conducta-, así como en el de expectativa, es decir, en el resultado que una persona espera tras llevar a cabo un cierto comportamiento. De acuerdo con este modelo, si los padres se perciben como personas capaces de explicar a sus hijos diversos aspectos relacionados con la sexualidad, es más probable que empiecen a conversar con ellos. De igual forma, cuando tienen la expectativa de obtener resultados favorables al hablar sobre sexualidad con sus hijos, tienen más probabilidades de entablar esas conversaciones; por el contrario, evitarán tales conversaciones cuando sus expectativas son negativas.

Las investigaciones sobre este tópico indican que los padres de hijos sin discapacidad platican con ellos sobre sexualidad cuando asocian dicha acción con consecuencias positivas, como evitar que sus hijos sufran embarazos no deseados; en cambio, los padres que evitan abordar dichos temas suelen pensar en que tratarlos tendrá consecuencias negativas para aquellos (Álvarez-Gayou, 2007). En el caso de los padres cuyos hijos tienen discapacidad, la relación entre esas variables es semejante, aunque en tales casos influye la preocupación de que sean víctimas de abuso sexual, además de que consideran que esos temas son demasiado complejos para su grado de entendimiento; al igual de lo que ocurre con los padres de hijos sin discapacidad, suelen evitar este tipo de conversaciones cuando creen que con ellas incitarán a sus hijos a tener relaciones sexuales (Bárcena y Rugerio, 2017).

Los conceptos de autoeficacia y de expectativas están fundamentados en la teoría del aprendizaje social (Bandura, 1977) y en la teoría social cognitiva (Bandura, 1986), las cuales han hecho posible el desarrollo de distintos programas de intervención orientados a promover la comunicación sobre la sexualidad entre los padres de hijos sin discapacidad (Dancy, Crittenden y Talashek, 2006; Dilorio, McCarty, Resnicow, Lehr y Denzmore, 2007; Kirby et al., 2004; Lederman, Chan y Roberts-Gray, 2008). Los referidos programas se caracterizan por proporcionar a los padres información sobre temas relevantes para los adolescentes, desarrollar en ellos habilidades de comunicación mediante el uso de técnicas de juego de roles, e implementar tareas para garantizar que conversen con sus hijos de manera efectiva. Los resultados indican que esta forma de intervención favorece la comunicación de los padres con sus hijos sobre tópicos sexuales, y también propicia que se sientan más seguros y cómodos al hacerlo.

Se han reportado diversas experiencias exitosas en el caso de los programas destinados a la educación sexual de jóvenes con discapacidad intelectual y la de sus padres (Alcedo, Aguado y Arias, 2006; Morentin, Arias, Rodríguez y Aguado, 2006; Olivier, 2006; Pineda y Gutiérrez, 2009). Sin embargo, esos programas se han desarrollado en países como España y Cuba, y si bien han utilizado algunas técnicas de probada eficacia (como el juego de roles), no se han centrado específicamente en el desarrollo de autoeficacia en los padres, ni tampoco en la generación de sus expectativas positivas hacia la comunicación con sus hijos. 
En las instituciones mexicanas de atención a usuarios de educación especial, es importante llevar a cabo programas dirigidos a capacitar a los padres para que proporcionen educación sexual a sus hijos. Como señalan Gil, Díaz, Ceccato, Ba1lester, y Giménez (2014) y Morell et al., 2017), muchos padres muestran actitudes más conservadoras que los profesionales que laboran en esas instituciones; además, suelen tener conocimientos muy escasos sobre las prácticas sexuales seguras y las de riesgo, o sobre cómo prevenir el abuso y la violencia sexual hacia sus hijos. Tales problemas pueden minimizarse si se abren espacios de discusión que les permitan desarrollar expectativas positivas y las habilidades de comunicación necesarias para educar sexualmente a sus hijos.

Para cumplir con dicho objetivo, un programa destinado a los padres ha de ir más allá de ser un mero espacio de discusión y análisis colectivo, toda vez que ha de considerar un conjunto de lineamientos, como los apuntados por Morentin et al. (2006) y Guevara et al. (2016): 1) Ser coherente con la perspectiva actual sobre la discapacidad y asumir un enfoque positivo de la sexualidad, incluyendo el énfasis en el bienestar, la calidad de vida y la autonomía, y no solamente enfocarse en cómo evitar y prevenir infecciones, embarazos y abusos; 2) Propiciar la comunicación entre padres e hijos, pues las interacciones familiares suelen ser los únicos recursos educativos disponibles para estas personas sobre la sexualidad; 3) Transmitir información científicamente comprobada que rompa con prejuicios y mitos; 4) Contar con un sistema de evaluación antes y después de la intervención que haga posible verificar los efectos del programa; 5) Utilizar recursos psicopedagógicos para que sea un programa estructurado y sistemático; 6) Presentar la información de manera ágil mediante la utilización de recursos técnicos y en términos accesibles a fin de que sea comprendida por personas de distintos niveles educativos, $y$ 7) Utilizar técnicas y dinámicas psicológicas para promover la motivación y la participación activa de los padres, pero sobre todo para desarrollar habilidades específicas para dialogar y educar sexualmente a sus hijos discapacitados.

Con base en lo anterior, la presente investigación tuvo como objetivo evaluar la efectividad de un programa de intervención fundamentado en los conceptos de autoeficacia y aprendizaje estructurado, dirigido a padres de jóvenes con discapacidad intelectual, para promover la comunicación sobre la sexualidad.

\section{MÉTODO}

\section{Participantes}

El grupo de participantes estuvo conformado por cuatro madres y un padre, con una edad promedio de 43 años, de los cuales cuatro estaban casados; la mayoría contaba con estudios de licenciatura, con un ingreso mensual se ubicó entre $\$ 6,749$ y $\$ 12,172$; todos se dedicaban al hogar y tres de ellos estaban jubilados. Las familias de los participantes estaban integradas por cuatro miembros (los padres y dos hijos). Los criterios para la inclusión de participantes fueron que sus hijos fueran discapacitados intelectualmente y tuvieran una edad de entre 12 y 29 años.

En cuanto a las características de los hijos, tres de ellos padecían síndrome de Down y dos más discapacidad intelectual de otro origen. En todos los casos, la discapacidad se había detectado desde su nacimiento. Tres eran mujeres y dos varones, con edades de entre 13 y 29 años, con un promedio de 18 años (D.E. $=3$ ).

\section{Diseño}

Se empleó un diseño $n=1$, que consiste en medir los cambios efectuados por cada participante después de la aplicación de un tratamiento, por lo que cada uno funge como su propio control (Kerlinger y Lee, 2002). Se hizo una evaluación antes de la intervención, otra inmediatamente después de la misma, y una tercera a los tres meses de haber concluido el programa.

\section{Instrumentos}

Se diseñó un conjunto de preguntas cuyo fin era obtener información de los padres respecto a las siguientes características sociodemográficas: sexo, edad, escolaridad, estado civil, ocupación, religión, ingreso mensual y número de hijos. También se recabó información sobre las características de 
los hijos, como sexo, edad, escolaridad, tipo de discapacidad y origen de la misma. Se incluyó una lista de cotejo que contenía un listado de dieciséis habilidades conductuales en la que los padres ubicaron las que sus hijos ya habían desarrollado, como vestirse, bañarse, comer, ir al baño, cocinar, convivir con otras personas, leer, escribir, sumar, restar, barrer, lavar trastes, tender la cama, salir a lugares cercanos, usar transporte y trabajar.

Se utilizó un instrumento de autorreporte, conformado por diez reactivos, para conocer las expectativas de los padres respecto a la comunicación con sus hijos sobre diversas cuestiones sexuales; seis de los reactivos correspondían a expectativas positivas (por ejemplo, "Si hablo con mi hijo[a] sobre temas de sexualidad puedo evitar que sufra abuso sexual"), y los cuatro reactivos restantes correspondían a expectativas negativas (por ejemplo, "Si hablo con mi hijo sobre la sexualidad lo incitaré a tener relaciones sexuales"), siendo sí o no las opciones de respuesta. El instrumento mostró un índice de consistencia interna de 0.85 .

También se incluyó una pregunta relativa a la manera en la cual los padres se comunicaban con sus hijos, cuyas opciones de respuesta fueron las siguientes: a) "De manera verbal, podemos mantener una conversación"; b) "Principalmente con frases cortas, pues tiene un lenguaje limitado"; c) "Con gestos, pues no habla"; d) "Con Lengua de Señas Mexicana", y e) "Otras". Además, con el propósito de obtener información sobre la autoeficacia percibida de los padres para hablar con sus hijos sobre diversos tópicos sexuales, se les preguntó si se sentían seguros de hablar con sus hijos sobre diecisiete temas específicos, como los cambios físicos que ocurren durante la adolescencia, qué es el noviazgo o cómo usar un condón. Cada pregunta se contestaba con sí o no. La variable de comunicación sobre sexualidad se obtuvo al preguntarles a los participantes si alguna vez habían hablado con sus hijos sobre cada uno de esos temas, siendo las opciones de respuesta las mismas que en el caso anterior. Por último, se obtuvo un indicador numérico con la suma del número de temas que habían abordado con sus hijos. Dichos instrumentos obtuvieron índices de consistencia interna de 0.87 y 0.89 , respectivamente.

\section{Procedimiento}

A través de redes sociales se hizo una invitación a diferentes grupos de asociaciones e instituciones que atienden a personas con discapacidad mediante un tríptico, el cual contenía información sobre los contenidos y duración del taller, así como las fechas y el lugar donde se efectuaría. Los asistentes al mismo recibieron y firmaron un formato de consentimiento informado en el que se establecía el carácter confidencial de la información recabada y el carácter voluntario de su participación. Luego, se les aplicó un instrumento de autoinforme para obtener información sobre las variables referidas, para lo cual la facilitadora explicó las instrucciones para responder el instrumento y aclaró las dudas de los padres de familia. Los participantes respondieron el instrumento en un tiempo de 20 minutos en promedio.

El programa de intervención se denominó ¿Cómo hablar de sexualidad a mi hijo con discapacidad? y estuvo compuesto por diez sesiones distribuidas semanalmente y con una duración de dos horas cada una, sesiones que se llevaron a cabo los días viernes de 10 a 12 horas en las instalaciones de la Facultad de Estudios Superiores Iztacala de la Universidad Nacional Autónoma de México, de la cual se obtuvo previamente el aval de su Comisión de Ética.

El objetivo general del taller estuvo encaminado a proporcionar conocimientos y habilidades a los padres para hablar con sus hijos sobre los ya citados diecisiete temas referentes a la sexualidad, y estuvo fundamentado en el aprendizaje estructurado propuesto por Goldstein (1973), referido a la conjunción sistematizada de cuatro técnicas para el cambio conductual: el modelamiento, el juego de roles (ensayo conductual), la retroalimentación (reforzamiento social) y el entrenamiento para la transferencia. De acuerdo a esta aproximación, es importante, en primer lugar, brindar a los participantes información sobre los temas de interés, y también acompañar dicha información con actividades que garanticen el desarrollo de habilidades conductuales. Por ello, en todas las sesiones se proporcionó a los padres la información mediante exposiciones y videos, acompañando la información con diversas estrategias, como preguntas de 
comprobación y actividades a realizar, con el fin de garantizar que los asistentes hubieran asimilado correctamente la información. Después se modelaba la manera en la cual podían entablar una conversación con sus hijos sobre cada tema, recurriendo para ello al uso de diversos materiales didácticos y videos; de este modo, además de los recursos verbales, los padres tenían a su disposición otros elementos de apoyo para transmitir la información a sus hijos.

La elección de los videos se basó en que tuvieran las características siguientes: ser de corta duración (menos de cinco minutos), emplear un lenguaje sencillo y mostrar los personajes en caricatura; se buscó privilegiar el canal visual, con imágenes que facilitaran la adquisición de la información, pero también se emplearon materiales como plastilina, penes de unicel para la enseñanza y tarjetas con imágenes, los cuales son fácilmente manipulables. Hecho lo anterior, los padres llevaban a cabo un juego de roles en el que ponían en práctica los comportamientos modelados previamente, recibiendo enseguida la retroalimentación por su ejecución. Al final de cada sesión, se les asignaban tareas cuyo propósito era que replicaran las conductas entrenadas en la sesión, pero esta vez con sus hijos y en su hogar. En la siguiente sesión, los padres describían su experiencia con la tarea encomendada y se les proporcionaba nuevamente retroalimentación. Tanto las actividades en el taller como las tareas tenían un carácter lúdico para favorecer la participación y la transmisión de la información. La organización del taller se presenta en la Tabla 1.

Tabla 1. Resumen de las sesiones del programa de intervención.

\begin{tabular}{|c|c|c|c|}
\hline $\begin{array}{c}N^{\circ} \\
\text { de sesión }\end{array}$ & Objetivos específicos & Actividades & Metodología \\
\hline 1 & $\begin{array}{l}\text { Aplicación del instrumento } \\
\text { de evaluación. } \\
\text { Adquirir conocimientos } \\
\text { y habilidades para } \\
\text { implementar estilos } \\
\text { de supervisión y apoyo } \\
\text { parental que favorezcan } \\
\text { el desarrollo de habilidades } \\
\text { en sus hijos mediante } \\
\text { el análisis de tareas. }\end{array}$ & $\begin{array}{l}\text { Sesión de evaluación (pretest). } \\
\text { Dinámica de presentación. } \\
\text { Introducción al concepto de discapacidad } \\
\text { intelectual (DI). } \\
\text { Información sobre supervisión y apoyo } \\
\text { parental, con énfasis en evitar } \\
\text { la sobreprotección. } \\
\text { Identificación de las actividades } \\
\text { que se dificultan a sus hijos. } \\
\text { Presentación del análisis de tareas como } \\
\text { estrategia para el desarrollo de habilidades } \\
\text { conductuales de sus hijos. } \\
\text { Elaboración de un análisis de tareas. }\end{array}$ & $\begin{array}{l}\text { Explicación de las instrucciones } \\
\text { del instrumento. } \\
\text { Exposición del tema. } \\
\text { Modelamiento para implementar } \\
\text { un análisis de tareas. } \\
\text { Elaboración de un análisis de tareas } \\
\text { para enseñar a sus hijos a elaborar } \\
\text { alimentos sin coacción. } \\
\text { Tarea: aplicar el análisis de tareas } \\
\text { en casa con sus hijos. }\end{array}$ \\
\hline 3 & $\begin{array}{l}\text { Adquirir habilidades } \\
\text { y estrategias para } \\
\text { comunicarse con sus hijos. }\end{array}$ & $\begin{array}{l}\text { Presentación acerca de las barreras } \\
\text { en la comunicación con sus hijos. } \\
\text { Presentación sobre la comunicación } \\
\text { asertiva. } \\
\text { Entrenamiento en habilidades } \\
\text { de comunicación. }\end{array}$ & $\begin{array}{l}\text { Modelamiento, juego de roles, } \\
\text { retroalimentación. } \\
\text { Tarea: tener una conversación } \\
\text { con sus hijos, empleando para ello } \\
\text { las estrategias vistas en la sesión. }\end{array}$ \\
\hline
\end{tabular}




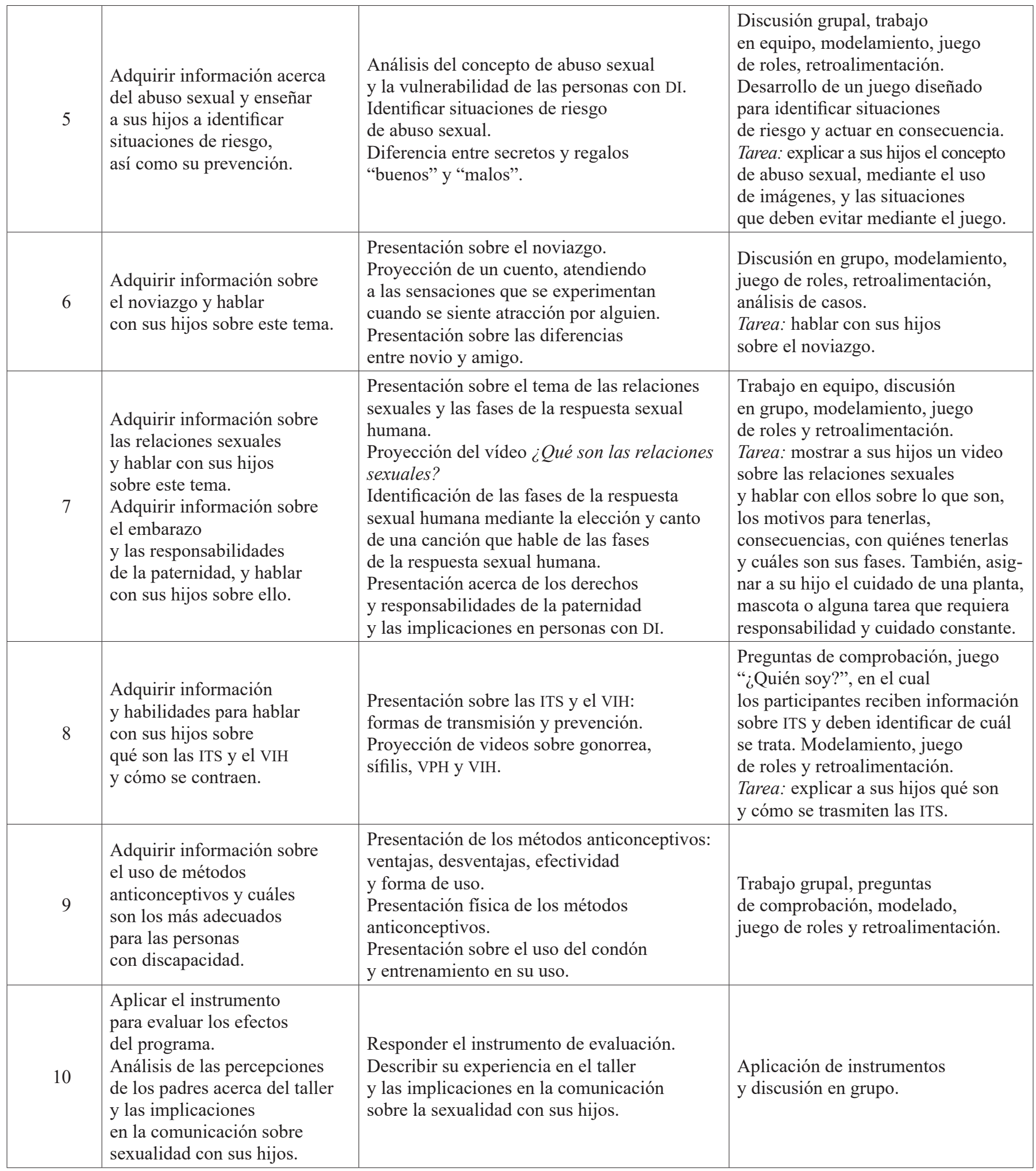

\section{RESULTADOS}

Por último, se aplicó nuevamente el instrumento de evaluación a todos los participantes tres meses después de haber concluido la intervención.
Se exponen a continuación los resultados más importantes. En primer lugar, se presentan los relacionados con la variable de expectativas, seguidos de los resultados de autoeficacia y comunicación 
sobre sexualidad; por último, se muestran las habilidades conductuales desarrolladas por los hijos de los participantes antes, después y luego de tres meses de concluida la intervención.

\section{Expectativas}

Los resultados sobre las expectativas se muestran en la Tabla 2. Como se puede observar, desde la fase de pretest la mayoría de los participantes reportaron tener expectativas positivas hacia la comunicación, y solo dos respondieron que consideraban muy difícil hablar de sexo con sus hijos (expectativas negativas). Después de la intervención, todos los padres reconocieron los efectos positivos de tal comunicación, y ninguno consideró que fuera difícil.

Tabla 2. Expectativas.

\begin{tabular}{|l|c|c|c|c|c|c|}
\hline \multicolumn{1}{|c|}{ Reactivo } & \multicolumn{2}{c|}{ Pre } & \multicolumn{2}{c|}{ Post } & \multicolumn{2}{c|}{ Seg. } \\
\hline Expectativas positivas & Sí & No & Sí & No & Sí & No \\
\hline E1. Si hablo con mi hijo(a) sobre la sexualidad sé que me entenderá. & 5 & 0 & 5 & 0 & 5 & 0 \\
\hline $\begin{array}{l}\text { E2. Si hablo con mi hijo(a) sobre la sexualidad puedo evitar que tenga } \\
\text { un embarazo no deseado. }\end{array}$ & 4 & 1 & 5 & 0 & 5 & 0 \\
\hline $\begin{array}{l}\text { E3. Si hablo con mi hijo(a) sobre la sexualidad puedo evitar que se contagie } \\
\text { de VIH o de alguna infección de transmisión sexual. }\end{array}$ & 4 & 1 & 5 & 0 & 5 & 0 \\
\hline $\begin{array}{l}\text { E4. Si hablo con mi hijo(a) sobre la sexualidad puedo evitar que sufra } \\
\text { abuso sexual. }\end{array}$ & 4 & 1 & 5 & 0 & 5 & 0 \\
\hline E5. Si hablo con mi hijo(a) sobre la sexualidad él(ella) me respetará. & 4 & 1 & 4 & 1 & 4 & 1 \\
\hline E6. Si hablo con mi hijo(a) sobre la sexualidad sentiré que hice lo correcto. & 5 & 0 & 5 & 0 & 5 & 0 \\
\hline Expectativas negativas & & & & & \\
\hline $\begin{array}{l}\text { E7. Hablar con mi hijo(a) sobre la sexualidad lo(la) incitará a tener } \\
\text { relaciones sexuales. }\end{array}$ & 0 & 5 & 0 & 5 & 0 & 5 \\
\hline E8. Si hablo con mi hijo(a) sobre la sexualidad podría sentirme apenado(a). & 0 & 5 & 0 & 5 & 0 & 5 \\
\hline E9. Hablar con mi hijo(a) sobre la sexualidad creo que será muy difícil. & 2 & 3 & 0 & 5 & 0 & 5 \\
\hline E10. Sin importar lo que le diga a mi hijo(a), él(ella) hará lo que quiera. & 1 & 4 & 1 & 4 & 1 & 4 \\
\hline
\end{tabular}

\section{Autoeficacia}

La Figura 1 ilustra los resultados en la variable de autoeficacia. Desde la preevaluación, los participantes reportaron que se sentían muy seguros de hablar con sus hijos sobre la mayoría de los temas sexuales, y solo uno de ellos dijo no sentirse seguro de hablar con su hijo sobre la masturbación. Durante el postest y en el seguimiento todos afirmaron estar seguros de hablar con sus hijos sobre todos los temas.

Figura 1. Número de padres que dijo sentirse seguro (autoeficacia) de hablar con sus hijos sobre la sexualidad en cada una de las fases del estudio.

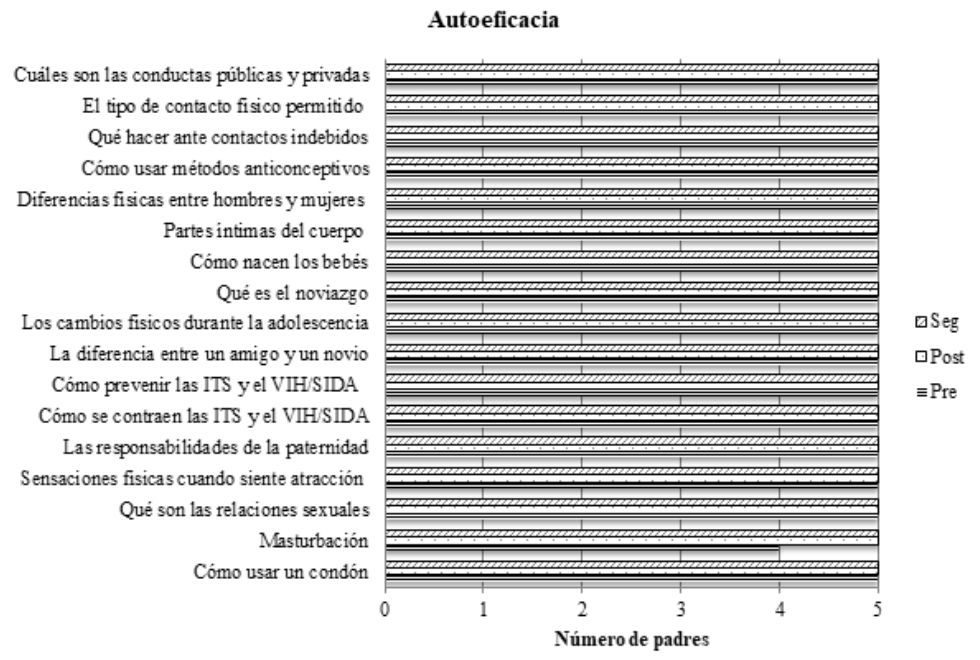




\section{Comunicación sobre sexualidad}

En la Figura 2 se muestra el número de participantes que afirmó hablar con sus hijos sobre cada uno de los temas evaluados durante el pretest, el postest y el seguimiento. Ya antes de la intervención algunos padres habían hablado con sus hijos sobre algunas cuestiones, principalmente las vinculadas a las conductas públicas y privadas, los tipos de contacto físico permitido y las partes íntimas del cuerpo; sin embargo, temas como las relaciones sexuales y la prevención de las ITS y el VIH habían sido tratados solamente por uno o dos participantes, y ninguno de ellos había abordado lo referente a la masturbación y el uso del condón. Después de la intervención, los cinco participantes reportaron hablar con sus hijos sobre la mayoría de los temas, excepto sobre cómo nacen los niños y la diferencia entre amigos y novios. En la fase de seguimiento todos los padres afirmaron mantener comunicación con sus hijos sobre todos los temas abordados en el taller.

Figura 2. Número de padres que reportaron hablar con sus hijos sobre los diferentes temas de la sexualidad durante cada fase del estudio.

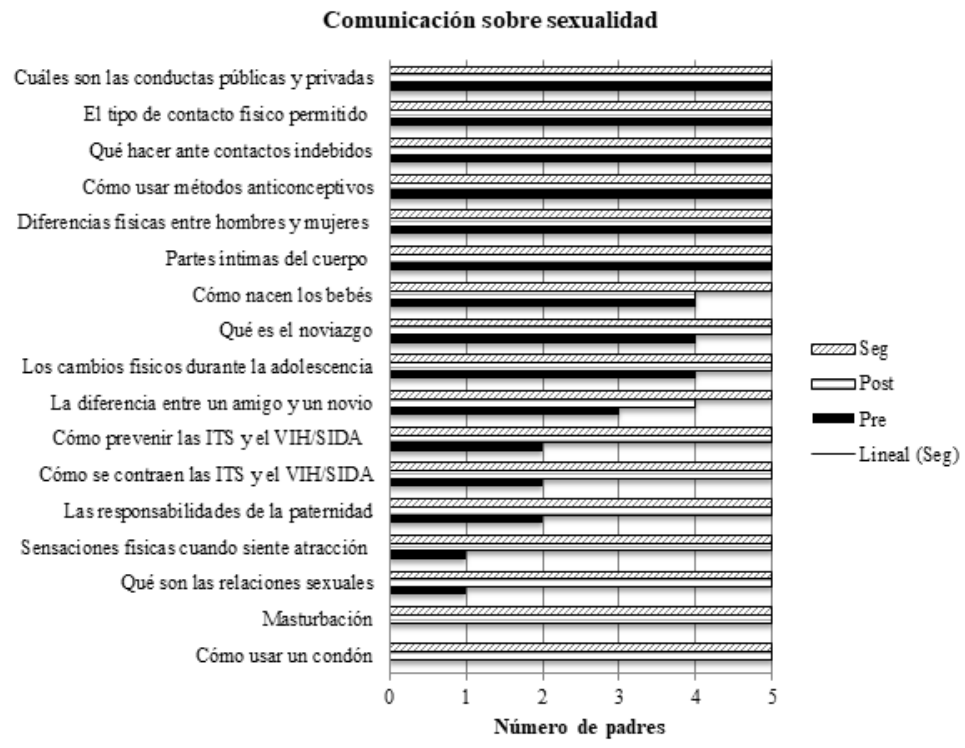

El número de temas que cada participante dijo abordar con su hijo en cada una de las fases del estudio aparece en la Figura 3.
Como puede apreciarse, todos los participantes aumentaron significativamente el número de temas abordados entre la preevaluación y la

Figura 3. Número de temas sobre la sexualidad abordados por cada participante con su hijo durante las tres fases del estudio.

Comunicación sobre sexualidad

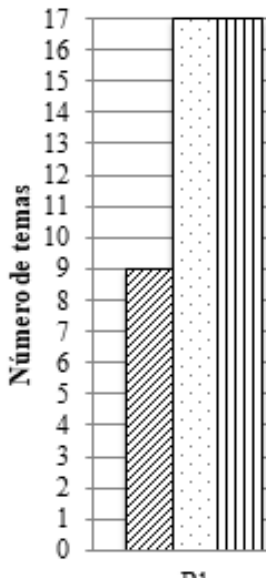

P1

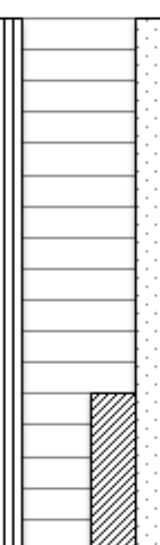

P2

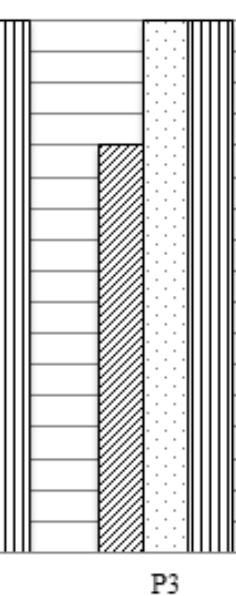

P3

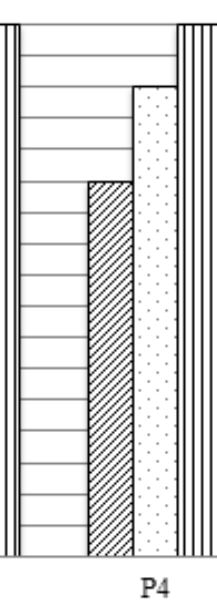

P4

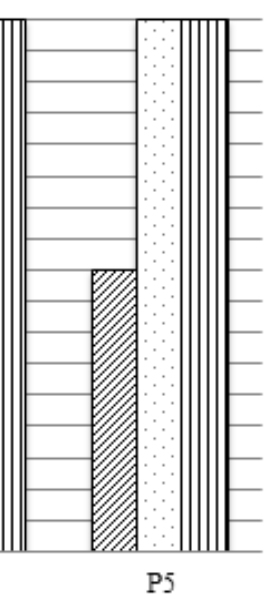

$\triangle$ Pre

$\square$ Post

凹Seg.

Participantes 
postevaluación, y en el seguimiento dijeron haber tratado con sus hijos todos los temas relacionados con el programa de intervención. Los participantes 1,2 y 5 fueron quienes reportaron conversar sobre un menor número de temas con sus hijos antes de la intervención, por lo que en ellos pueden ubicarse los mayores efectos del programa.

\section{Habilidades conductuales desarrollas por los hijos de los participantes}

Como muestra la Figura 4, durante la evaluación inicial todos los participantes aseveraron que sus hijos sabían vestirse, comer, ir al baño y convivir con otras personas; solo uno de los jóvenes no era capaz de bañarse de manera independiente, tres de ellos sabían leer y restar, y cuatro podían hacer sumas; cuatro sabían lavar los trastes, tres podían barrer y dos tendían su cama; ninguno cocinaba ni usaba transportes, y solo uno trabajaba y salía sin compañía a lugares cercanos. En la fase de seguimiento tres de los padres reportaron que sus hijos habían aumentado el número de las habilidades conductuales desarrolladas; en el caso del 1 y el 5, la habilidad adquirida fue la de tender la cama; en el caso del número 3, el joven adquirió dos habilidades: bañarse y barrer.

Figura 4. Número de habilidades conductuales desarrolladas por los hijos de los participantes en cada fase del estudio.

Habilidades conductuales desarrolladas

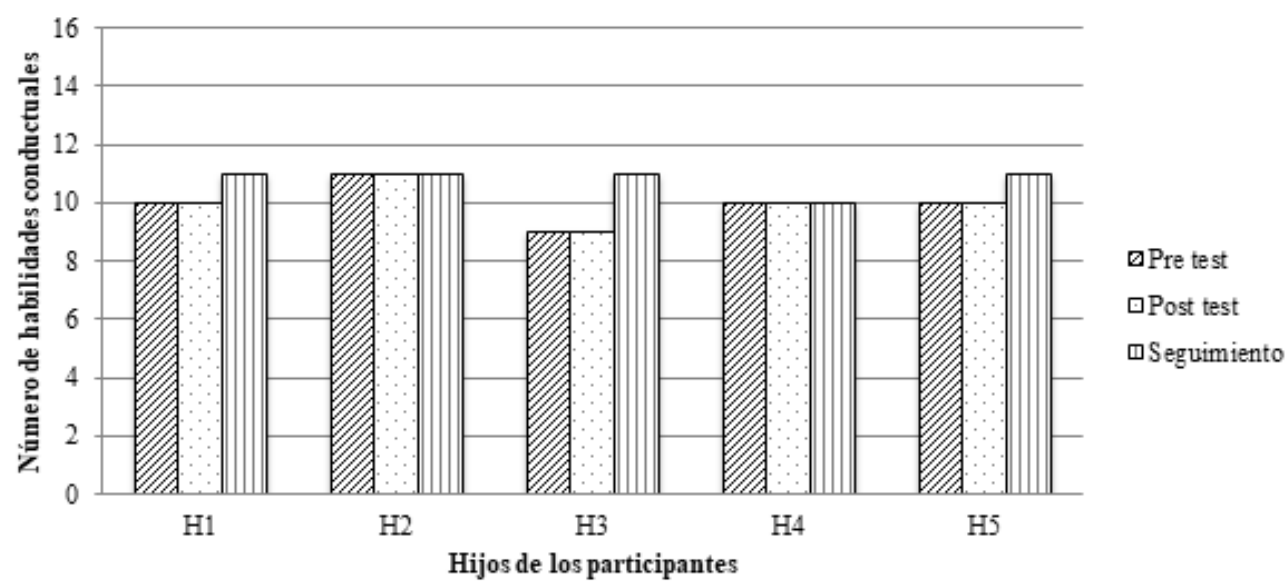

\section{DISCUSIÓN}

Dado que el propósito de este estudio fue evaluar los efectos de un programa de intervención, fundamentado en los conceptos de autoeficacia y aprendizaje estructurado, y dirigido a padres de hijos con discapacidad intelectual para promover la comunicación con sus hijos sobre temas de sexualidad, los resultados más importantes indican que el programa resultó efectivo para dicho fin. A continuación se discuten los aspectos más relevantes relacionados con tales resultados.

En primer lugar, es necesario subrayar que solamente fueron cinco los participantes, quienes compartían algunas características sociodemográficas, tales como un nivel educativo profesional, dedicación al hogar y pertenencia a familias con- formadas por cuatro integrantes (dos padres y dos hijos). Respecto a las características de sus hijos, la mayoría contaba con estudios de secundaria, y todos habían desarrollado un repertorio de habilidades conductuales suficientes para desempeñarse adecuadamente en las tareas hogareñas y escolares. Es muy probable que esas características de unos y otros favorecieran la disposición a participar en el taller y lograr sus efectos positivos. Ello lleva necesariamente a reconocer que el programa puede obtener resultados distintos al aplicarse a poblaciones con características diferentes porque, de acuerdo a Bárcena, Guevara y Álvarez (2017), el grado de comunicación entre padres e hijos discapacitados sobre la sexualidad se relaciona con factores tales como el número de integrantes de la familia y el nivel de estudios de los padres, así 
como con las características de los jóvenes, como su nivel de estudios, la cantidad de habilidades conductuales desarrolladas, el tipo de discapacidad y el origen de la misma, entre otros.

Sin embargo, dado que el programa se basa en un modelo sistemático de aprendizaje estructurado, y está centrado en la autoeficacia y las expectativas positivas, hay razones para suponer que puede ser efectivo al aplicarse a padres con características distintas, o cuyos hijos tengan otro nivel de desarrollo o un tipo diferente de discapacidad. Desde luego, en tales casos deberán hacerse algunas adecuaciones, como por ejemplo el tipo de materiales a utilizar por parte de los padres cuando sus hijos manifiestan deficiencias auditivas o visuales. Además, es posible que en el trabajo con padres con un menor nivel educativo o con ideas menos abiertas hacia la comunicación sobre temas sexuales deba diseñarse un mayor número de sesiones de intervención, e incluso planear un modo de difusión distinto para convocarlos al taller y motivarlos para participar en él. Al respecto, debe admitirse que la respuesta a la convocatoria relacionada con este trabajo fue muy pobre. En el futuro se espera replicar este estudio, aunque ahora con un mayor número de participantes. Por ahora, lo importante es haber generado un programa dirigido a la promoción de la salud de los jóvenes con discapacidad, cuyo acceso a los programas preventivos es sumamente limitado (OMS, 2015).

Ahora bien, es posible afirmar que los resultados del programa de intervención descrito fueron positivos en diversos aspectos. Por un lado, se logró un impacto directo en la comunicación porque todos los padres participantes lograron entablar un diálogo con sus hijos e impartirles enseñanzas sobre todos los temas de sexualidad revisados, lo que fue posible porque se emplearon recursos didácticos (videos y materiales didácticos) durante la intervención, pero sobre todo porque se utilizaron técnicas de exposición, modelamiento, juego de roles, retroalimentación y entrenamiento para la transferencia, cuyo efecto en los padres fue el desarrollo de sus habilidades de comunicación. Si se considera que la autoeficacia descansa en las experiencias de éxito y fracaso, es probable que el hecho de que los padres lograran practicar dichas habilidades en el taller les hizo sentirse más segu- ros para abordar los temas con sus hijos, tal como lo demuestran los resultados de este estudio.

Otro aspecto que indica resultados favorables del programa se relaciona con las expectativas de los padres acerca de la comunicación sobre la sexualidad con sus hijos. Desde la preevaluación, algunos participantes reportaron tener ciertas expectativas positivas, sobre todo en cuanto a evitar el abuso sexual hacia sus hijos, aunque dos padres dijeron que ello sería muy difícil. Durante el taller se les proporcionó información sobre las consecuencias positivas de la comunicación con sus hijos sobre la sexualidad, así como de los efectos negativos de no tenerla; además, se diseñaron actividades orientadas a promover en ellos ideas positivas sobre tal comunicación. Estas estrategias lograron que las expectativas positivas aumentaran significativamente y que disminuyeran las negativas en la fase de postest, resultados que se mantuvieron hasta la fase de seguimiento.

En lo cuanto a los temas de sexualidad abordados por los padres en la comunicación con sus hijos, antes de la intervención informaron tratar solamente temas como la prevención del abuso, las conductas públicas y privadas y los cambios físicos que ocurren en la adolescencia, pero ninguno sobre la masturbación, las ITS, el VIH o el uso del condón. En la fase de seguimiento, no obstante, se cumplieron esos objetivos porque los cinco padres de familia hablaron con sus hijos sobre los diecisiete temas referentes a la sexualidad, muy probablemente como efecto del programa, que los sensibilizó para abordar dichos temas en casa.

Como dato adicional, cabe señalar que el programa también tuvo un efecto positivo en el desarrollo de las habilidades de los hijos de los participantes. Antes de empezar la intervención, los jóvenes contaban con algunas habilidades conductuales, como vestirse, comer, ir al baño y convivir con otras personas (con excepción de uno, que no era capaz de bañarse solo); después del taller, sin embargo, tres de los jóvenes aumentaron su repertorio al poder ya tender la cama, bañarse y barrer. Es posible explicar este resultado a partir de haber diseñado la intervención para promover el desarrollo de habilidades mediante el uso del análisis de tareas. Con ello se atiende, al menos en parte, el problema de la sobreprotección, tan 
frecuente en las prácticas de crianza de las personas con discapacidad intelectual (Caricote, 2012); también se hizo posible un mayor grado de autonomía y privacidad en los jóvenes, así como una mayor participación en la toma de decisiones en el seno familiar, aspectos esenciales para su desarrollo psicológico, incluida su autoestima, según los señalamientos de Björnsdóttir et al. (2017). De hecho, es importante sugerir que estudios futuros se encaminen expresamente a analizar estos aspectos trabajando con las prácticas de crianza y los patrones interactivos en las familias de personas con discapacidad.

En resumen, el taller ¿Cómo hablar de sexualidad a mi hijo con discapacidad? logró resultados favorables y cumplió con sus objetivos. Cabe enfatizar que si bien los padres de familia iniciaron el taller con expectativas positivas y altos reportes de autoeficacia, la intervención mantuvo su interés, promovió cambios en quienes no se sentían seguros de hablar sobre la sexualidad con sus hijos, y generó cambios favorables en las capacidades comunicativas de los padres. El ambiente establecido durante las sesiones del taller propició el intercambio de ideas entre ellos, reduciendo las actitudes negativas hacia la sexualidad de las personas discapacitadas, lo que es un aspecto esencial para el desarrollo psicosexual de las mismas (Morell et al., 2017).
Para concluir, es importante reconocer que las limitaciones del presente estudio, relacionadas con el número y características de los participantes, así como el hecho de no haber evaluado los estilos de crianza paternos, restringen la generalización de los hallazgos toda vez que pueden determinar en gran medida el tono, la forma y la efectividad de la comunicación entre padres e hijos.

Por tales razones, es deseable emprender estudios con muestras mayores y más heterogéneas, así como evaluar los estilos de crianza y otros factores que pueden afectar la comunicación. De igual manera, es importante incorporar al taller a los hijos, y comparar qué forma de aplicación del programa es más efectiva para su educación sexual. Otros aspectos que pueden incluirse en los programas de intervención para maximizar su impacto son, a saber: la evaluación y desarrollo de competencias para la conducta sexual-amorosa de las personas con discapacidad, las actividades dirigidas a la prevención de la violencia sexual y otras formas de maltrato, así como diversos tópicos relacionados con la formación integral de estos jóvenes (López, 2014). Lo importante es hallar las mejores estrategias disponibles para esta población, dado el alto riesgo de que sufra abuso sexual, ITS, VIH/sida y embarazos no deseados, tal como han documentado diversos estudios (Giménez et al., 2017; Morell et al., 2017; Robles y Guevara, 2017).

\section{REFERENCIAS}

Alcedo, M., Aguado, A. y Arias, B. (2006). Eficacia de un programa de educación sexual en jóvenes con discapacidad intelectual. Análisis y Modificación de la Conducta, 142(32), 217-239.

Álvarez-Gayou, J.L. (2007). Educación de la sexualidad: ¿en la casa o en la escuela? Los géneros, la escuela y la educación profesional de sexualidad. México: Paidós.

Bandura, A. (1977). Social learning theory. New York: General Learning Press.

Bandura, A. (1986). Social foundations of thought and action: A social cognitive theory. Englewood Cliffs, NJ: Prentice Hall.

Bárcena G., S.X., Guevara B., Y. y Álvarez B., E, (2017). Características de padres e hijos con discapacidad que intervienen en la comunicación sobre sexualidad. Journal of Behavior, Health \& Social Issues, 9(2), 36-44. doi: 10.1016/j.jbhsi.2018.01.002.

Bárcena, S., Robles, S. y Díaz-Loving, R. (2013). El papel de los padres en la conducta sexual de sus hijos. Acta de Investigación Psicológica, 3(1), 956-968.

Bárcena, S. y Rugerio, J.P. (2017). Expectativas vinculadas a la comunicación sobre sexualidad en padres de hijos con discapacidad. Memorias del XXXVI Congreso Interamericano de Psicología, Mérida, Yuc. (México), 23-27 de julio.

Björnsdóttir, K., Stefánsdóttir, Á. y Valgerður, G. (2017). People with intellectual disabilities negotiate autonomy, gender and sexuality. Sexuality and Disability, 35(3), 295-311.

Caricote, E. (2008). Influencia de los padres en la educación sexual del adolescente. Educere, 12(40), 79-87.

Caricote, E. (2012). Sexualidad en adolescentes discapacitados. Salus, 16(2), 53-57. 
Chen, J., Dunne, M.P. y Han, P. (2007). Prevention of child sexual abuse in China: Knowledge, attitudes, and communication practices of parents of elementary school children. Child Abuse and Neglect, 31(7), 747-755. doi: 10.1016/j.chiabu.12.013.

Dancy, B.L., Crittenden, K.L., y Talashek, M.L. (2006). Mothers' effectiveness as HIV risk reduction educators for adolescent daughters. Journal of Health Care Poor Underserved, 17, 218-239.

Díaz, I., Gil, M., Ballester, R., Morell, V. y Molero, R. (2014). Conocimientos, comportamiento y actitudes sexuales en adultos con discapacidad intelectual. International Journal of Developmental and Educational Psychology, 3(1), 415-422.

DiIorio, C., Dudley, W.N., Wang, D.T., Wasserman, J., Eichler, M., Belcher, L. y West-Edwards, C. (2001). Measurement of parenting self-efficacy and outcome expectancy related to discussions about sex. Journal of Nursing Measurement, 9(2), 135-149.

Dilorio, C., McCarty, F., Resnicow, K., Lehr, S. y Denzmore, P. (2007). Real men: A group-randomized trial of an HIV prevention and intervention for adolescent boys. American Journal of Public Health, 97(6), 1084-1089.

Eisenberg, M.E., Sieving, C., Bearinger, L.H., Swain, C. y Resnick, M.D. (2006). Parental notification laws for minors' access to contraception -What do parents say? Archives of Pediatrics \& Adolescent Medicine, 159, 120-125.

García P., C., Loredo A., A., Trejo, J. y Casas, A. (2013). Violación sexual en niños y adolescentes: una urgencia médica. Acta Pediátrica de México, 34(5), 288-294.

Gil L., M., Díaz R., I., Ceccato, R., Ballester A., R. y Giménez G., C. (2014). Conocimientos, actitudes hacia la orientación sexual, comportamientos de riesgo y abusos sexuales en mujeres con discapacidad intelectual. International Journal of Developmental and Educational Psychology, 2(1), 397-405.

Giménez G., C., Gil L., M., Ruiz P., E. y Díaz R., I. (2017). Abuso sexual y discapacidad intelectual: cómo identifican y valoran la experiencia las personas con discapacidad intelectual y los profesionales que les atienden. International Journal of Developmental and Educational Psychology, 4(1), 129-136.

Goldstein, A. (1973). Structured learning therapy: toward a psychotherapy for the poor. Nueva York: Academic Press.

Guevara, Y., Robles, S., Aguilar, Y. y Luna, N. (2016). Educación sexual en personas con discapacidad. Fundamentos para programas de intervención. México: Universidad Nacional Autónoma de México.

Guevara, Y., Robles, S., Rodríguez, M. y Frías, B. (2016). Comunicación con padres, comportamiento sexual, e indicadores de abuso sexual en jóvenes mexicanos con discapacidad visual. Psychologia: Avances de la Disciplina, 10(1), 79-90.

Howland, C.A., y Rintala, D.H. (2001). Dating behaviors of women with physical disabilities. Sexuality and Disability, 19, 41-70.

Insa B., E. (2005). El desarrollo de la sexualidad en la deficiencia mental. Revista Interuniversitaria de Formación del Profesorado, 19(3), 327-343.

Instituto Nacional de Salud Pública y Organización Mundial de la Salud (2016). Encuesta Nacional de Salud (ENSANUT). Cuernavaca (México): INSP/OMS. Recuperado de http://ensanut.insp.mx/informes/ENSANUT2012ResultadosNacionales2Ed.pdf.

Kerlinger, F.N. y Lee, H.B. (2002). Investigación del comportamiento. Métodos de investigación en ciencias sociales. México: McGraw-Hill.

Kijak, R. (2013). The sexuality of adults with intellectual disability in Poland. Sexuality and Disability, 31, 109-123. doi: 10.107/ s11195-013-9294-8.

Kirby, D.B, Baumler, E., Coyle, K.K., Basen-Engquist, K., Parcel, G.S, Harrist, R. et al. (2004). The "Safer Choices" intervention: Its impact on the sexual behaviors of different subgroups of high school students. Journal Adolescent Health, 35, 442-452.

Lederman, R.P., Chan, W. y Roberts-Gray, C. (2008). Parent-Adolescent Relationship Education (PARE): Program delivery to reduce risks for adolescent pregnancy and STDs. Journal of Behavioral Medicine, 33, 137-143.

López, F. (2014). Abusos sexuales y otras formas de maltrato sexual: Intervenciones educativas y teapéuticas. Madrid: Síntesis.

Markham, C.M., Lormand, D., Gloppen, K.M., Peskin, M.F., Flores, B., Low, B. y House, L.D. (2010). Connectedness as a predictor of sexual and reproductive health outcomes for youth. Journal of Adolescent Health, 46(3), S23-S41.doi: 10.1016/j. jadohealth.2009.11.214.

Morell M., V., Gil L., M., Díaz R., I. y Caballero G., L. (2017). Actitudes de padres, profesionales y población general hacia la sexualidad de las personas con discapacidad física e intelectual. International Journal of Developmental and Educational Psychology, 4(1), 173-183.

Morentin, R., Arias, B., Rodríguez, J. y Aguado, A. (2006). Pautas para el desarrollo de programas eficaces de educación afectivo-sexual en personas con discapacidad intelectual. Revista Española sobre Discapacidad Intelectual, 37(217), 41-58.

Nosek, M., Foley, C., Hughes, R. y Howland, C. (2001). Vulnerabilities for abuse among women with disabilities. Sexuality and Disability, 19(3), 177-189. doi:10.1023/A:1013152530758.

Olivier, S. (2006). Educación sexual para personas con discapacidad intelectual. Desicio, 19(1), 54-59.

Organización Mundial de la Salud (2015). Discapacidad y salud. Ginebra: OMS. Recuperado de http://www.who.int/mediacentre/ facts $\neg$ heets/fs352/es/.

Organización Mundial de la Salud (2016). Adolescentes: riesgos para la salud y soluciones. Ginebra: OMS.

Pereda, N. (2016). ¿Uno de cada cinco?: victimización sexual infantil en España. Papeles del Psicólogo, 37(2), 126-133. 
Pineda, E. y Gutiérrez, E. (2009). Estrategia de intervención educativa sobre la sexualidad en niños con síndrome de Down. Revista Cubana de Medicina General Integral, 25(3), 95-104.

Rivera, P. (2008). Sexualidad de los niños, niñas y jóvenes con discapacidad. Revista Educación, 32(1), 157-170.

Robles S., S. y Guevara, Y. (2017). Comportamientos de riesgo y abuso sexual en varones y mujeres con discapacidad visual o auditiva. Revista Española de Discapacidad, 5(I), 133-151. doi: 10.5569/2340-5104.05.01.08.

Rodríguez, Y., Aguilar, B. y García, I. (2012). Consecuencias psicológicas del abuso sexual infantil. Eureka, 9(1), 58-68.

Schaalma, H.P., Abraham, C., Gilmore, M.R. y Kok, G. (2004). Sex education as health promotion: “What does it take?”. Archives of Sexual Behavior, 33(3), 259-269.

Shakespeare, T. (2000). Disabled sexuality: Towards rights and recognition. Sexuality and Disability, 18, 159-166.

Smith, D. (2008). Disability, gender and intimate partner violence: Relationships from the behavioral risk factor surveillance system. Sexuality and Disability, 26(1), 15-28. doi: 10.1007/s11195-007-9064-6. 